\title{
Análise do impacto das Interface Gestuais em Softwares de Geometria Interativa no desempenho dos alunos
}

\author{
Helena Macedo Reis (helenamcd@fatectq.edu.br) \\ Vitória Otrenti Frade (vitoria.of.559@gmail.com) \\ Ramílio Ramalho Reis Filho (ramilio.filho@fatectq.edu.br) \\ Faculdade de Tecnologia de Taquaritinga - FATEC-TQ \\ Taquaritinga, Brasil
}

\begin{abstract}
Resumo. A interface é o principal mecanismo de interação entre os usuários e as funcionalidades do sistema. No contexto educacional, interfaces desenvolvidas de forma apropriada podem minimizar os esforços dos estudantes a aprender sobre as características da interface e direcionar sua atenção no aprendizado da geometria. Estudos indicam que interfaces que dispõe de vários elementos em tela podem prejudicar no processo de ensino-aprendizagem, em que o estudante pode sentir-se confuso em escolher qual funcionalidade deseja. Como proposta de solução deste problema, pesquisadores propõe o uso de interfaces gestuais, em que há redução do número de elementos em tela, fazendo com que os estudantes possam direcionar sua atenção ao aprendizado da geometria. Entretanto, não há estudos empíricos que evidenciam este beneficio. Desta forma, este artigo apresenta os resultados de um experimento no qual investigou-se como a gestão da memória de trabalho e conhecimento em geometria pode influenciar no treinamento de usuários aprendendo a utilizar um software de geometria interativa por gestos. Os resultados experimentais não indicaram diferenças significativas entre os estudantes em relação aos escores obtidos nos testes.
\end{abstract}

Palavras-chave: geometria interativa, teoria da carga cognitiva, interface gráfica do usuário

\section{Impact analysis of Gesture Interfaces for Interactive Geometry Software in student performance}

Abstract. The interface is the main mechanism of interaction between users and system functionality. In the educational context, interfaces developed properly can minimize the efforts of students to learn about the interface features and direct their attention on learning geometry. Studies indicate that interfaces that show several elements can harm the process of teaching and learning, in which students may feel confused to choose what functionality they want to use. As a proposal solution of this problem, researchers proposed the use of gestural interfaces, where there is reduction in the number of elements on the interface, so that students can direct their attention to geometry learning. However, there is no empirical studies that show this benefit. Thus, this article presents the results of an experiment in which investigated the management of working memory and knowledge in geometry can influence the user training using an interactive geometry software for gestures. The experimental results indicated no significant differences between the students in relation to the scores obtained in the tests.

Keywords: interactive geometry, cognitive theory load, graphical user interface 


\section{Introdução}

O computador digital tem sido empregado no ensino, praticamente, desde seu surgimento em 1945, mas é a partir da década de 80, com o aparecimento dos computadores pessoais (personal computers), os PCs, que a utilização desta máquina e de seus recursos (software) provocaram grande impacto na educação (Kortenkamp, 2010). O uso de ferramentas computacionais na educação é importante, desde que dispõem recursos diferenciados, como o uso de interfaces específicas para cada atividade. Diante de dificuldades com o aprendizado, muitos educadores vêm incorporando o uso de ferramentas computacionais para auxiliar no ensino.

Dentre essas ferramentas computacionais apresentadas na forma de aplicativos gráfico, aparecem os sistemas de Geometria interativa (SGI), que têm como objetivo permitir aos estudantes aprenderem geometria utilizando o computador, por meio da manipulação dinâmica de objetos geométricos, como, por exemplo, retas, circunferências e pontos (Isotani e Brandão 2004; Erez e Yerushalmy 2007; Roanes-Lozano 2003). Os SGIs simplificam a abordagem do conteúdo para o estudo de geometria, fazendo com que o usuário perceba que utilizando ferramentas tradicionais, como transferidor, régua $\mathrm{e}$ compasso, gastaria muito mais tempo.

Apesar dos benefícios, a interface do SGI pode atrapalhar no processo de ensinoaprendizagem da geometria. A quantidade de ícones/botões apresentados em tela pode apoiar os usuários experientes, entretanto, os iniciantes podem ficar confusos em escolher a funcionalidade desejada. Isto acontece devido a necessidade de o usuário memorizar do que se trata de cada função de determinado ícone, fazendo com que o usuário consuma grande parte de sua carga cognitiva para o aprendizado da interface do SGI ao invés da geometria. A carga cognitiva refere-se às cargas colocadas na memória de trabalho do aprendiz durante a instrução (Reis et al., 2012; Borges et al., 2013; Borges et al., 2015).

Para evitar que essa frustração aconteça, é necessário reduzir a quantidade de elementos, tornando a interface mais simples e amigável, respeitando a capacidade de memorização de cada usuário. Uma proposta de solução seria o uso de interfaces gestuais, em que o usuário pode construir os objetos geométricos por meio de gestos, que são considerados mais naturais e que necessitam de menos tempo de treinamento (Hanne, 1994).

Portanto, os autores deste trabalho vêm investigando como as interfaces gestuais do SGIs afetam a aprendizagem e como elas podem ser adaptadas para atender as necessidades dos alunos. Este experimento envolveu 21 alunos e 2 instrutores, utilizando um software de geometria interativa denominado Sketchometry. Duas habilidades foram avaliadas: $(i)$ conhecimento em geometria e a (ii) capacidade da memória de trabalho. Estas competências foram selecionadas, pois é de interesse dos autores identificar o grau de influência destas habilidades em usuários não treinados, utilizando um SGI pela primeira vez.

Para tal, na seção 2 são apresentados os trabalhos relacionados. Na seção 3 são apresentadas brevemente sobre interfaces de SGIs, teoria da carga cognitiva e memória de trabalho. As seções 4 e 5 descrevem o experimento elaborado e os resultados. E finalmente, nas seções 6 e 7 temos as ameaças à validade e a conclusões da pesquisa realizada, respectivamente. 


\section{Trabalhos Relacionados}

Atualmente é de nosso conhecimento a existência de quatro sistemas que possibilitam o aprendizado da geometria por meio de gestos: (a) Geometry $\mathrm{Pad}^{*}$, (b) GeoGebra ${ }^{\dagger}$, (c) Sketchometry ${ }^{\ddagger}$ e (d) GeoTouch ${ }^{\S}$. Apesar de existirem estudos empíricos referente à usabilidade da interface destes sistemas (Reis et al., 2014; Reis et al., 2015), nenhum deles investigou o uso do sistema em sala de aula, envolvendo alunos. Os estudos empíricos realizados envolveram somente especialistas em usabilidade, utilizando as dez heurísticas de Nielsen (1993) para avaliar as interfaces.

Os softwares Geometry Pad e Geogebra possuem interfaces semelhantes. Ambos possuem um menu inferior, por onde os usuários podem escolher as funcionalidades que desejam executar, sendo semelhante as interfaces que possuem interação por meio de clique/mouse.

Em relação ao Sketchometry e Geotouch, suas interfaces possuem menos botões/ícones apresentados em tela. Ambos possuem menu, entretanto é direcionado somente para acessar funcionalidades básicas do software (i.e. abrir documento, novo documento). Apesar dos dois softwares possuírem interfaces semelhantes (e.g. redução de ícones em tela) e construção dos objetos geométricos por meio de gestos, a interação é executada de forma diferente. Enquanto no Sketchometry o usuário pode fazer construções sem conhecimentos sólidos em geometria, no Geotouch, o usuário deve conhecer todos os conceitos das propriedades geométricas para realizar a construção dos objetos geométricos. Por exemplo, para construir uma circunferência, no Sketchometry o usuário pode fazer somente o gesto de circunferência. No Geotouch, o usuário deve ter conhecimento que toda circunferência parte de um centro, sendo desta forma, necessário que o usuário construa um ponto central e depois faça o movimento de circunferência.

\section{Interface dos SGIs, Teoria da Carga Cognitiva e Memória de Trabalho}

Há vários estudos que investigaram o impacto das interfaces de SGIs na carga cognitiva dos usuários (Reis et al., 2012; Borges et al., 2013; Borges et al., 2015). Os resultados sugerem que interfaces que possuem várias informações apresentadas em tela podem fazer com que o uso da carga cognitiva seja direcionada a aprender a interface do sistema ao invés da geometria. A carga cognitiva faz parte da teoria da carga cognitiva (TCC) que consiste na capacidade do ser humano em processar muitas informações ao mesmo tempo. $\mathrm{O}$ acúmulo dessas informações acaba dificultando o entendimento do conteúdo que tal pessoa planeja aprender. Pois acaba exigindo um esforço excessivo para todo esse procedimento cognitivo, ocasionando uma sobrecarga da mente.

A TCC divide a carga cognitiva dentro de três diferentes tipos: intrínseca, persistente e estranha (Schimpf \& Spannagel, 2011). A carga cognitiva intrínseca se refere a complexidade do conteúdo da aprendizagem. A carga cognitiva persistente se refere as atividades que favorecem o propósito do aprendizado. Já a carga cognitiva estranha diz respeito a todo conteúdo das atividades que não está ligado a aprendizagem de modo direto.

Combinada com o uso da carga cognitiva, a memória de trabalho também pode ser afetada pela interface do SGI. Existem dois tipos de memória, a de curto prazo e a de

\footnotetext{
${ }^{*}$ http://www.stemonmobile.com/geometry-pad/

${ }^{\dagger}$ http://www.geogebra.org/cms/pt_BR/download/

$\$$ http://www.sketchometry.org

$\S$ http://goo.gl/5Oacf5
} 
longo prazo. A memória de curto prazo (memória de trabalho), diz respeito ao armazenamento temporário de dados com a capacidade limitada. Já a memória de longo prazo, possui uma capacidade mais ampliada e um armazenamento de dados mais organizados.

Portanto, como uma possível solução para interfaces que apresentam vários ícones/botões dispostos em tela seria o uso das interfaces gestuais. Estas interfaces podem fazer que seja reduzido o número de elementos em tela, buscando eliminar o uso da carga cognitiva estranha. Além disso, espera-se que os usuários possam memorizar rapidamente os gestos, por serem naturais e que permaneçam na memória de longo prazo.

\section{Experimento: Design e Execução}

Apesar de existirem estudos (Reis et al., 2012; Borges et al., 2013; Borges et al., 2015) que investigam a quantidade de elementos das interfaces dos SGIs afetam no processo de ensino-aprendizagem da geometria, ainda não há nenhum estudo empírico que envolve o uso das interfaces gestuais e o seu impacto com alunos. Somente há estudos que avaliam a interface por meio de Heurísticas (Nielsen, 1992) por parte de especialistas em usabilidade. Deste modo, este experimento foi planejado com o propósito de averiguar maneiras mais eficientes de distribuir os sujeitos da pesquisa em grupos balanceados a fim de se realizar novamente os testes com diferentes versões de SGI de interfaces gestuais e compará-los com as versões de SGI que possuem interação por meio de clique/mouse. Este experimento foi baseado no experimento realizado por Borges et al. (2012), seguindo sua metodologia.

\subsection{Planejamento}

Esta seção apresenta o planejamento usado no experimento com o objetivo de investigar uma possível relação entre as variáveis independentes como capacidade da (i) memória de trabalho e (ii) geometria dos usuários e seu desempenho resolvendo exercícios de geometria utilizando um sistema de geometria interativa em interfaces gestuais. As seguintes questões de pesquisa $(\mathrm{QP})$ foram elaboradas:

QP1: Em termos de usabilidade, a capacidade de memorização por parte dos usuários influencia no aprendizado do software Sketchometry?

QP2: Em termos de usabilidade, o grau de proficência dos usuários em Geometria influencia no aprendizado do software Sketchometry?

No experimento, a usabilidade foi definida a partir das notas obtidas pelos sujeitos ao resolverem a lista de exercícios utilizando o software Sketchometry pela primeira vez e sem treinamento prévio. Para a execução do experimento, os seguintes passos foram executados: (i) questionário de questões pessoais, (ii) teste de conhecimentos de geometria e (iii) teste para avaliar a capacidade da memória de trabalho. O experimento foi dividido em duas partes, sendo a primeira, os sujeitos preencheram e solucionaram os três primeiros itens, e na segunda parte, resolveram a lista de exercícios utilizando o Sketchometry.

\subsubsection{Definição dos objetivos}

As metas do experimento foram baseadas no paradigma Goal/Question/Metric (CGM), sugerida por Wohlin et al., 2000). As metas foram organizadas em cinco partes, sendo: Objeto de Estudo, Objetivos, Perspectiva, Foco Qualitativo e Contexto.

Objeto de estudo: A interface de usuário de um software de Geometria Interativa chamado Sketchometry 
Objetivo: Avaliar a influência das variáveis independentes $(i)$ conhecimento em geometria, e (ii) capacidade de memória de trabalho na utilização de uma GUI para um SGI. O aprendizado de um software pode demandar considerável consumo de carga cognitiva do aprendiz. Usuários que possuem alta capacidade de utilização de suas memórias de trabalhos, podem não ter dificuldades em aprender a interface do software e conseguem focar no aprendizado da geometria. Entretanto, usuários que possuem menor capacidade de manipulação da memória de trabalho podem demorar mais tempo em aprender a utilizar o software, fazendo com que o aprendizado seja focado na interface ao invés da geometria.

Perspectiva: $\mathrm{O}$ experimento será executado do ponto de vista dos pesquisadores

Foco Qualitativo: O efeito primário investigado é a influência das variáveis independentes na utilização da $G U I$ do Sketchometry mesurada pelas notas obtidas pelos sujeitos nos testes e questionários propostos.

Contexto: Estudantes de Graduação em Sistemas de Internet com pouco ou nenhum conhecimento prévio sobre SGIs.

O experimento pode ser sumarizado pelo modelo abaixo (Wohlin et al., 2000):

Analisar a GUI do software Sketchometry

para o propósito de avaliar

com respeito à usabilidade

do ponto de vista de pesquisadores

no contexto de estudantes de Graduação em Sistemas de Internet com pouca ou nenhuma experência utilizando SGIs

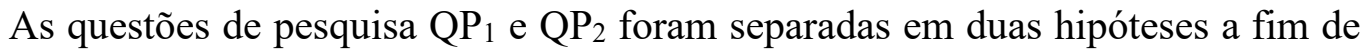
viabilizar a aplicação de testes estatísticos. Cada hipótese foi testada em busca de evidências da possível correlação linear entre as variáveis independentes e a pontuação obtida pelos sujeitos utilizando o SGI. O primeiro par de hipóteses recebeu o nome de Memória de Trabalho, é identificada pelo acrônimo MT e se relaciona com $\mathbf{Q P}$.

Hipótese Nula, MTo: Não há relação linear significativa entre a capacidade da memória de trabalho dos usuários e o aprendizado do Sketchometry (em termos das notas obtidas pelos sujeitos na resolução dos exercícios). Formalmente: MT0: $\mathbf{r}=\mathbf{0}$

Hipótese alternativa, MT1: Existe uma relação linear significativa entre a capacidade da memória de trabalho dos usuários e o aprendizado do Sketchometry (em termos das notas obtidas pelos sujeitos na resolução do teste). Formalmente: $\mathbf{M T}_{\mathbf{1}}: \mathbf{r} \neq \mathbf{0}$

O segundo par recebeu o nome de Conhecimento em Geometria (CG) e se relaciona $\operatorname{com} \mathbf{Q P}_{2}$.

Hipótese Nula, CG0: Não há relação linear significativa entre o conhecimento de Geometria dos usuários e o aprendizado do Sketchometry (em termos das notas obtidas pelos sujeitos na resolução dos exercícios). Formalmente: CG0: $\mathbf{r}=\mathbf{0}$

Hipótese alternativa, CG1: Existe uma relação linear significativa entre o conhecimento de Geometria dos usuários e o aprendizado do Sketchometry (em termos das notas obtidas pelos sujeitos na resolução do teste). Formalmente: $\mathbf{C G}_{1}$ : $\mathbf{r} \neq \mathbf{0}$

\subsection{Design do Experimento}

Para verificar as hipóteses, aplicou-se um modelo com fatores simples, com somente um tratamento para cada variável. As principais variáveis independentes foram memória de 
trabalho (MT) e conhecimento em geometria (CG) que foram comparados, alternadamente, à principal variável dependente que é a nota obtida pelos sujeitos ao resolver uma lista de exercícios utilizando o SGI. Esta principal variável foi denominada Sketchometry. Com o objetivo de mensurar as habilidades dos sujeitos, foram aplicados testes. O teste de geometria consistiu de 11 questões de múltipla escolha, desde o nível elementar até questões que demandavam maior conhecimento. A cada questão correta foi atribuído o valor de 0,91 pontos.

O teste de memória de trabalho utilizado emprega a técnica conhecida como $n$ back test (Dehn, 2008). Foi utilizado o site Cognitive Fun que disponibiliza gratuitamente softwares que permitem realizar o $n$-back test. $\mathrm{O}$ teste consiste na apresentação de uma sequência de imagens, uma a uma, durante um período de tempo. O sujeito realizando o teste, é repetida e sequencialmente, convidado a responder se a figura que ele está visualizando no presente momento é igual a figura que foi apresentada " $\mathrm{n}$ " figuras anteriores. $\mathrm{O}$ tempo de resposta é contabilizado no teste, quanto maior o resultado do 2back test, pior o desempenho do sujeito, e vice-versa. A Figura 1 apresenta as etapas do experimento:

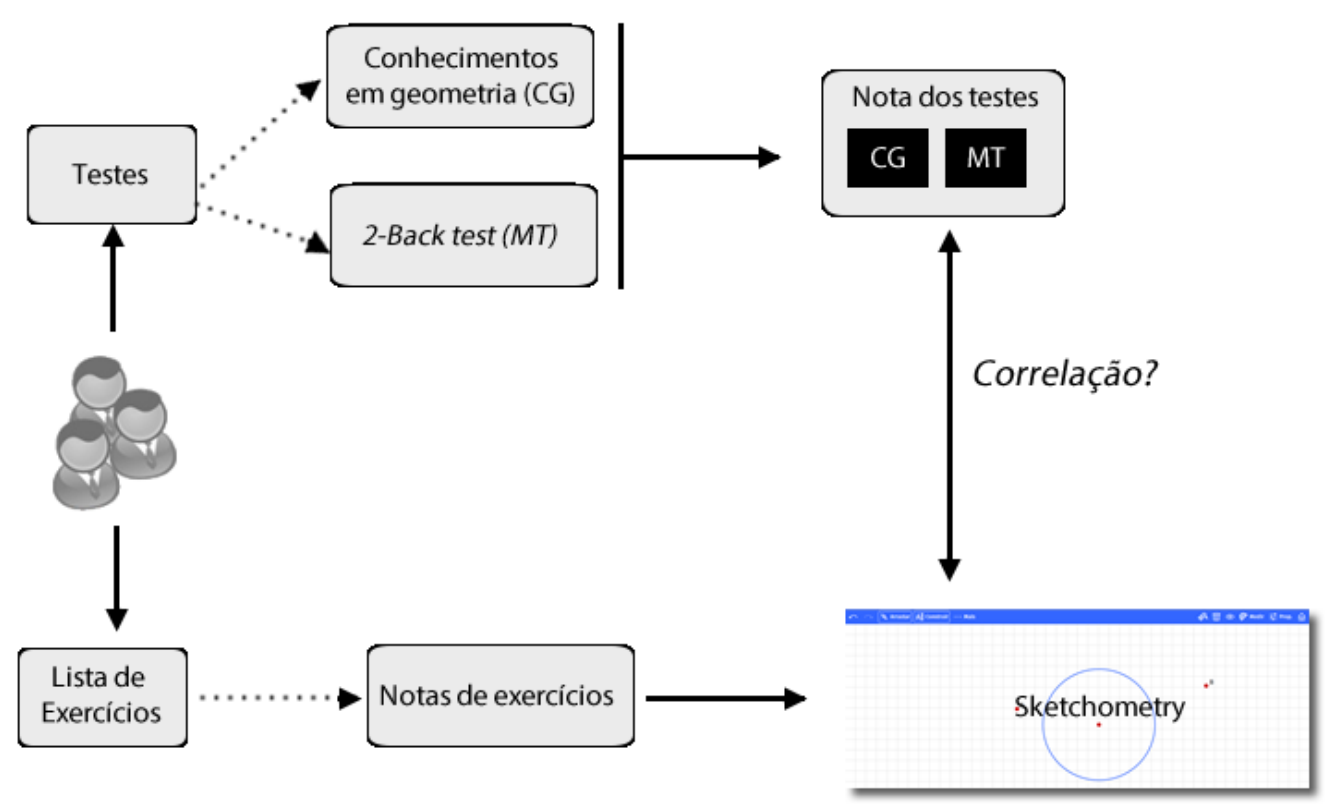

Figura 1. Etapas da execução do experimento

A Tabela 1 apresenta a pontuação dos sujeitos nos três testes. A Tabela 2 sumariza a pontuação apresentada na Tabela 1. Uma análise inicial dos resultados indica que apenas 4 estudantes solucionaram corretamente menos de $50 \%$ dos exercícios propostos. Outros 17 alunos restantes conseguiram atingir resolver entre 50\% a 95\% dos exercícios propostos.

Tabela 1. Pontuação dos sujeitos nos testes

\begin{tabular}{|c|c|c|c|}
\hline Sujeito & MT & CG & Sketchometry \\
\hline A & 1,01 & 6,37 & 6,00 \\
\hline B & 1,69 & 7,28 & 7,50 \\
\hline C & 1,63 & 4,55 & 8,50 \\
\hline D & 1,58 & 2,73 & 8,50 \\
\hline
\end{tabular}




\begin{tabular}{|c|c|c|c|}
\hline E & 1,18 & 5,46 & 8,50 \\
\hline F & 1,80 & 4,55 & 9,50 \\
\hline G & 2,26 & 6,37 & 6,50 \\
\hline H & 1,68 & 4,55 & 2,00 \\
\hline I & 9,26 & 5,46 & 8,50 \\
\hline J & 1,68 & 8,19 & 8,00 \\
\hline K & 2,78 & 9,10 & 8,50 \\
\hline L & 3,35 & 3,64 & 8,00 \\
\hline M & 1,15 & 4,55 & 6,00 \\
\hline N & 8,23 & 6,37 & 5,00 \\
\hline O & 2,15 & 0,91 & 9,50 \\
\hline P & 1,80 & 6,37 & 1,00 \\
\hline Q & 11,00 & 3,64 & 3,00 \\
\hline R & 1,17 & 7,28 & 9,00 \\
\hline S & 1,14 & 6,37 & 7,00 \\
\hline T & 20,35 & 5,46 & 3,00 \\
\hline U & 2,03 & 8,19 & 9,00 \\
\hline
\end{tabular}

Tabela 2. Estatística descritiva sumarizando a pontuação dos sujeitos nos testes

\begin{tabular}{|l|c|c|c|}
\hline & MT & CG & Sketchometry \\
\hline Mínimo & 1,01 & 0,91 & 1,00 \\
\hline Máxima & 20,35 & 9,10 & 9,50 \\
\hline Média & 3,76 & 5,59 & 6,79 \\
\hline Mediana & 1,80 & 5,46 & 8,00 \\
\hline Desvio Padrão & 4,75 & 1,96 & 2,57 \\
\hline
\end{tabular}

\section{Análise dos resultados}

Esta seção sumariza os resultados observados com base no que foi apresentado anteriormente. O coeficiente da correlação é uma medida da associação linear entre duas variáveis. O coeficiente de correlação utilizado foi o de Spearman, que é um teste de correlação não-paramétrico para determinar a intensidade com que duas variáveis estão associadas linearmente. $\mathrm{O}$ valor do coeficiente pode variar entre os valores -1 e 1 , onde 1 indica uma relação linear positiva perfeita, -1 representa uma relação linear negativa e o indica que não há nenhuma relação linear. Na Tabela 3 , a força da correlação é expressa usando o padrão "Guia Michelin", cuja a escala $(* \mathrm{p}<0,05$, significativo; ** valor de $\mathrm{p}<$ 0,01 , altamente significativo; $* * * p<0,001$, extremamente significativo). Quando não existe uma significância estatística, NS é usado e o valor p é dado entre parênteses. Os resultados foram agrupados de acordo com a questão de pesquisa.

Tabela 3. Estatística descritiva sumarizando os cálculos da correlação e da regressão

\begin{tabular}{|l|c|c|}
\hline & MT0, MT1 & CG0, CG1 \\
\hline r(Spearman) & $-0,117$ & $-0,006$ \\
\hline r2 & $-0,411(41 \%)$ & $0,019(19 \%)$ \\
\hline p-value & $\mathrm{NS}(0,615)$ & $\mathrm{NS}(0,979)$ \\
\hline
\end{tabular}


QP1 - De acordo com o valor da correlação $(r)$ entre MT e os resultados obtidos na utilização do Sketchometry, nota-se que, quanto menores os resultados obtidos em MT, maior foi a nota obtida pelo sujeito durante a utilização do Sketchometry. A correlação entre as variáveis é fraca e negativa. O valor obtido para $r 2$ indica que $41 \%$ da nota utilizando o Sketchometry possa ser explicada com a capacidade da memória de trabalho do sujeito (MT). Entretanto, não se pode rejeitar a hipótese nula $\left(\mathrm{MT}_{0}\right)$ porque o valor para o $\mathrm{p}$ ( $p$-value) é maior do que 0,05 .

QP2 - De acordo com o valor da correlação $(r)$ entre CG e os resultados obtido na utilização do Sketchometry, pode se notar que, aparentemente, que o conhecimento de geometria prévio não afeta diretamente no uso da ferramenta. De acordo com o valor calculado, a correlação entre as variáveis é fraca e negativa. $O$ valor obtido para $r 2$ indica que $19 \%$ da nota usando o Sketchometry pode ser explicada pelo conhecimento prévio em geometria por parte dos sujeitos (CG). Entretanto, não é possível rejeitar a hipótese nula $\left(\mathrm{CG}_{0}\right)$ porque o valor para o $\mathrm{p}$ ( $\mathrm{p}$-value) é maior que 0,05 .

Este resultado não era esperado, uma vez que se acreditava que quanto maior conhecimento em geometria maior seria a nota no Sketchometry. Atribuímos a instabilidade do resultado pelo fato do Sketchometry não relacionar os conceitos das propriedades geométricas para realizar a construção dos objetos geométricos. Por exemplo, os usuários que sabem os conceitos da reta paralela talvez não conseguiram realizar esta mesma construção no Sketchometry, pois o gesto para esta construção é em formato de "Z" e o software não possui material de ajuda.

Acredita-se que ao executar o experimento novamente com um número maior de sujeitos em um software em que os gestos possuem relação com conceitos da geometria, este fenômeno tende a não se repetir.

\section{Ameaças à Validade}

Uma possível ameaça a validade dos resultados é a representatividade das amostras. Todos os sujeitos que participaram do estudo são estudantes de graduação, portando não se pode garantir que conclusões semelhantes seriam obtidas a partir dos resultados se fosse utilizada uma população semelhante. Não é possível ter certeza que esses resultados possam ser generalizados para toda população de graduandos. Desta forma, sugere-se que haja repetições destes estudos em diferentes amostras da população. Além disso, existiu a preocupação em garantir o que foi avaliado em vista da usabilidade da GUI do Sketchometry, entretanto a falta de conhecimento em geometria de alguns participantes pode ter impedido que alguns completassem os exercícios propostos.

\section{Conclusão}

O dado que aparentemente exerce alguma influência no uso da interface por usuários iniciantes é a capacidade da memória de trabalho. Foi observado que $41 \%$ da nota utilizando o Sketchometry pode ter sido influenciada pelas habilidades de memorização dos usuários.

Aparentemente, o conhecimento prévio em geometria dos usuários não afeta na aprendizagem da ferramenta. Apesar de uma quantidade significativa de sujeitos que possuíram nota de geometria abaixo da média (11 sujeitos, representando 52\%), o uso da ferramenta não foi afetado, sendo que a maioria (13 sujeitos, representando 62\%) possuíram notas acima da média. Nenhum sujeito obteve nota máxima no teste de geometria e tampouco na lista de exercícios no Sketchometry. Por este motivo, para um próximo experimento planeja-se utilizar uma ferramenta que possua relação dos gestos para a construção dos objetos geométricos com as propriedades geométricas, como o 
Geotouch. Acredita-se que o conhecimento prévio de geometria pode influenciar na construção dos objetos geométricos, fazendo com que os usuários consigam aprender a interface em menos tempo.

Outro benefício deste experimento foi a disponibilização de um pacote de replicação ${ }^{* *}$ que possa ser utilizado por outros pesquisadores com os mesmos propósitos. Os testes estatísticos mostraram-se inconclusivos, não sendo possível rejeitar nenhuma das hipóteses nulas formuladas devido aos altos valores de $\mathrm{p}$ obtidos.

\section{Referências}

BORGES, S. S. ; REIS, H. M. ; MARQUES, L. B. ; DURELlI, VINICIUS H. S. ; BITTENCOURT, I. I. ; MAILLARD, P. A. J. ; ISOTANI, S. . Reduced GUI for Interactive Geometry Software: Does It Affect Students' Performance?. Computers in Human Behavior, v. 54, p. 124-133, 2015.

BORGES, S. S. ; REIS, H. M. ; MORO, L. F. S. ; DURELLI, V. H. S. ; ISOTANI, S. . Agrupamento Balanceado de Sujeitos a fim de Testar a Interface Gráfica de um Software de Geometria Interativa. RENOTE. Revista Novas Tecnologias na Educação, v. 11, p. 1-10, 2013.

EREZ, M. M.; YERUSHALMY, M. "If You Can Turn a Rectangle into a Square, You Can Turn a Square into a Rectangle ..." Young Students Experience the Dragging Tool. International Journal of Computers for Mathematical Learning, vol. 11, n. 3, páginas 271-299, 2007.

HANNE, K.-H. Multimodal Communication, Natural Language and Direct Manipulation (Gestures) in Human-Computer Interation. In: E DWARDS, A. D. N.; H OLLAND, S., eds. Multimedia Interface Design in Education, Capítulo: 11, NATO ASI Series,

página 223, 1994.

ISOTANI, S.; BRANDÃO, L. Ferramenta de avaliação automática no iGeom. In: Anais do Simpósio Brasileiro de Informática na Educação (SBIE), páginas 328-337, 2004.

KOTERNKAMP, U.; DOHRMANN, C. User interface design for dynamic geometry software. Acta Didactica Napocensia, 3(2). Available at http://dppd.ubbcluj.ro/adn/article_3_2_6.pdf. 2010

NIELSEN, J. Finding Usability Problems Through Heuristic Evaluation. In: In Proceedings of CHI '92 (Monterey), páginas 373-380, 1992.

NIELSEN, J.; MOLICH, R. Heuristic evaluation of user interfaces. In: CHI '90 Proceedings of the SIGCHI Conference on Human Factors in Computing Systems, páginas 249-256, 1990.

ROANES-LOZANO, E.; ROANES-MACÍAS, E.; VILLAR-MENA, M. A bridge between dynamic geometry and computer algebra. Mathematical and Computer Modelling, vol. 37, n. 9-10, páginas 1005-1028, 2003.

REIS, H. M. ; BORGES, S. S. ; ISOTANI, S. Análise de Usabilidade de Sistemas de Geometria Interativa para Tablets. RENOTE. Revista Novas Tecnologias na Educação, v. 12, p. 1-10, 2014.

REIS, H. M. ; ISOTANI, S. . Concepção de uma Família de Gestos para Construção de Objetos Geométricos e sua Utilização em um Sistema de Geometria Interativa para Dispositivos Móveis: GeoTouch. In: Congresso Brasileiro de Informática na Educação, 2015, Maceió. Anais do Concurso de Teses Dissertações e Trabalhos de Conclusão de Curso (CTD-IE), 2015. p. 1-10.

REIS, H. M. ; BORGES, S. S. ; DURELLI, V. H. S. ; MORO, L. F. S. ; BRANDAO, A. A. F. ; Ellen Francine Barbosa ; BRANDAO, L. O. ; MAILLARD, P. A. J. ; BITTENCOURT, I. I. ; ISOTANI, S. . Towards Reducing Cognitive Load and Enhancing Usability Through a Reduced Graphical User Interface for a Dynamic Geometry System: An Experimental Study.

** http://goo.gl/9qSy9f 
In: IEEE International Symposium on Multimedia, 2012, Irvine,CA. Proceedings of the IEEE International Symposium on Multimedia, 2012. p. 445-450.

SCHIMPF, F.; SPANNAGEL, C. Reducing the graphical user interface of a dynamic geometry system. ZDM, 43(3), 389-397. 2011.

WOHLIN, C.; RUNESON, P.; HÖST, M.; OHLSOON, M. C.; REGNELL, B.; WESSLÉN, A. Experimentation in Software Engineering: An Introduction. Kluwer Academic Publishers, 2000. 